\title{
Retención dentaria múltiple: Reporte de un caso y revisión de literatura
}

\author{
Multiple dental retention: Case report and literature review \\ Retenção dentária múltipla: Um relato de caso e revisão da literatura
}

Recibido: 29/04/2021 | Revisado: 03/05/2021 | Acepto: 09/05/2021 | Publicado: 27/05/2021

\author{
Paula Bernarda Proaño Benenaula \\ ORCID: https://orcid.org/0000-0001-9710-1497 \\ Universidad de Cuenca, Equador \\ E-mail: bernarda.proano08@ucuenca.edu.ecr \\ Johanna Monserrath Sáenz Delgado \\ ORCID: https://orcid.org/0000-0003-2006-3108 \\ Universidad de Cuenca, Equador \\ E-mail: johanna.saenz@ucuenca.edu.ec \\ Marcelo Enrique Cazar Almache \\ ORCID: https://orcid.org/0000-0002-6806-7442 \\ Universidad de Cuenca, Equador \\ E-mail: marcelo.cazar@ucuenca.edu.ec
}

\begin{abstract}
Resumen
La retención dental múltiple es una patología que presenta varios órganos dentales ubicados en el interior de los procesos alveolares tanto del maxilar como de la mandíbula. Teniendo en consideración que esta patología puede verse relacionada con factores locales o sistémicos que condicionan su presencia y puede estar acompañada de patologías adicionales como en este caso, por quistes dentígeros. A continuación, se reporta el caso de un paciente masculino de 20 años que presenta 13 piezas dentales retenidas, el cual posee una enfermedad sistémica no relacionada con dicha patología. El objetivo es informar e incentivar a realizar una correcta anamnesis, análisis clínico y radiológico detallado, que nos permitan llegar a un correcto diagnóstico y un buen plan de tratamiento; con la finalidad de generar una base para futuras investigaciones.
\end{abstract}

Palabras clave: Dientes impactados; Síndrome de dientes impactados múltiples; Dientes sin erupcionar.

\begin{abstract}
Multiple dental retention is a pathology that presents several dental organs located inside the alveolar processes of both the maxilla and the mandible. Taking into consideration that this pathology may be related to local or systemic factors that condition its presence and may be accompanied by additional pathologies, such as, in this case, dentigerous cysts. Next, the case of a 20 -year-old male patient with 13 retained teeth is reported, who has a systemic disease not related to said pathology. The objective is to inform and encourage a correct anamnesis, detailed clinical and radiological analysis, which allow us to reach a correct diagnosis and a good treatment plan; in order to generate a basis for future research.
\end{abstract}

Keywords: Impacted teeth; Multiple impacted teeth syndrome; Erupted teeth.

\section{Resumo}

A retenção dentária múltipla é uma patologia que apresenta vários órgãos dentais localizados dentro dos processos alveolares da maxila e da mandíbula. Levando em consideração que essa patologia pode estar relacionada a fatores locais ou sistêmicos que condicionam sua presença e pode estar acompanhada de patologias adicionais, como, neste caso, os cistos dentígeros. A seguir, é relatado o caso de um paciente do sexo masculino, 20 anos, com 13 dentes retidos, portador de doença sistêmica não relacionada à referida patologia. O objetivo é informar e estimular uma anamnese correta, análises clínicas e radiológicas detalhadas, que nos permitam chegar a um diagnóstico correto e a um bom plano de tratamento; a fim de gerar uma base para pesquisas futuras.

Palavras-chave: Dentes impactados; Síndrome dos dentes múltiplos impactados; Dentes erupcionados.

\section{Introducción}

La retención dental múltiple es una condición rara que describe a varias piezas dentarias ubicadas en el interior de los maxilares, las cuales son incapaces de erupcionar completamente, a pesar de llegar a su etapa normal de erupción; además 
mantienen la integridad de su saco pericoronario fisiológico (Ajith, Shetty, Hussain, Nagaraj, \& Srinath, 2014; Sosa Rosales et al., 2003; Yeman, 2003).

Este tipo de anomalía puede afectar a cualquier órgano dental ya sea permanente, supernumerario o temporal, siendo este último el de menor frecuencia de aparición (Delavarian, Delavarian, \& Imani, 2018). Comúnmente las piezas afectadas son los caninos superiores en aproximadamente 1 - $3 \%$ de la población (Conley et al., 2007; Uslu, Akcam, Evirgen, \& Cebeci, 2009); seguido de los terceros molares inferiores y superiores, los caninos inferiores y los segundos premolares (Casas Acosta et al., 2020). Además, algunos autores como Conley, Sreevedi y Manne señalan que hay mayor incidencia de aparición en el sexo femenino en comparación con el masculino (Ajith et al., 2014; Conley et al., 2007; Manne, Gandikota, Juvvadi, Rama, \& Anche, 2012).

En cuanto a la etiología se puede clasificar en dos grupos: factores sistémicos y locales. (Ajith et al., 2014; Bedoya \& Park, 2009; Conley et al., 2007)

Entre las enfermedades sistémicas o síndromes asociados encontramos trastornos genéticos como displasia cleidocraneal, síndrome de Gardner, osteopetrosis, hipotiroidismo, Síndrome de Zimmerman- Laband, y síndrome de Noonay (Choukroune, 2017; Delavarian et al., 2018; Reddy et al., 2018; Vani, Nooney, Raju, \& Hemadri, 2014). Estos afectan de manera generalizada a la mayor parte de la dentición, en comparación con los factores locales. (Casas Acosta et al., 2020; Vani et al., 2014)

Al mencionar las causas locales, son varias entre estas tenemos barreras físicas, falta de espacio (apiñamiento dental), posición anormal de la yema dental, alteraciones metabólicas locales, traumatismos, formación de quistes e infecciones. (Delavarian et al., 2018; Sujatha et al., 2012; Vani et al., 2014).

A continuación, se describe un caso clínico que reporta un paciente con discapacidad intelectual y epilepsia que presenta múltiples retenciones dentarias permanentes acompañado de la presencia de quistes dentígeros, además se analizarán ciertas características que deben ser analizadas y nos pueden dar pautas sobre un problema sistémico de base.

\section{Metodología}

El presente artículo tiene un enfoque metodológico cuantitativo, que consiste en el reporte de un caso clínico, el cual cumple con las normas éticas ya que se cuenta con una historia clínica detallada y los representantes del paciente firmaron un consentimiento informado debido a la condición de salud que padece el paciente; además recalcamos que se cuenta con la aprobación respectiva para su publicación respetando siempre el anonimato del paciente (Hernandez, 2018).

Los autores del artículo se autofinancian para este proyecto y cuentan con el apoyo del docente de la Facultad de Odontología de la Universidad de Cuenca.

\section{Presentación del Caso}

Paciente masculino de 20 años de edad, acude a la consulta privada acompañado por sus padres, pues necesitan plantear un posible tratamiento tomando en cuenta las piezas dentales presentes en boca. (Ilustración 1). En cuanto a sus antecedentes médicos, el paciente en cuestión presenta discapacidad intelectual originada por hipoxia fetal durante el momento del parto, de igual manera se ha diagnosticado epilepsia en el primer año de vida, señalando también dos crisis importantes que se observaron a los 6 y 17 años de edad. 
Ilustración 1. Fotografía frontal del paciente.

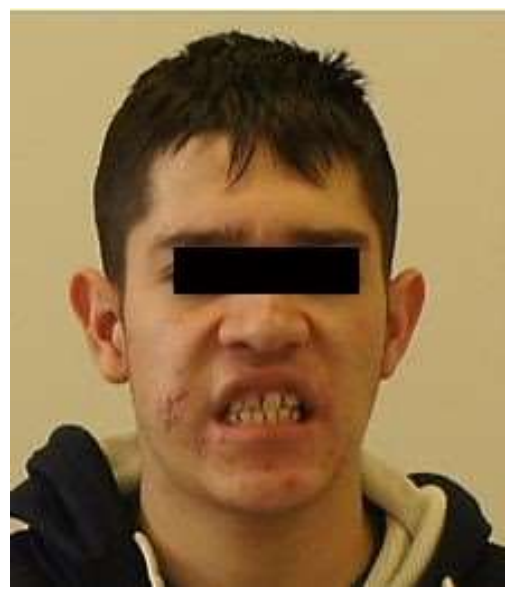

Fuente: Autores.

Luego de realizar una indagación en cuanto a antecedentes familiares se destaca que tanto a su familia materna y paterna, se ha visto afectada por la presencia de cuadros de epilepsia, al igual que antecedentes de cáncer estomacal y de piel. Con relación al tratamiento farmacológico del paciente se destacan dos fármacos antiepilépticos que son el Valpakine y Lamictal, señalando además alergia al fármaco Epamin.

Sus antecedentes odontológicos señalan la presencia de múltiples restauraciones dentales, exodoncias quirúrgicas de piezas inferiores por la presencia de quistes periapicales y la tracción dental de piezas anterosuperiores mediante ortodoncia fija durante 3 meses a la edad de 8 años.

Al examen clínico intraoral se puede observar, edentulismo parcial clase I de Kennedy a nivel mandibular y a nivel maxilar clase I de Kennedy con 1 modificación; además encontramos la presencia de 12 piezas dentales, divididos en 5 piezas dentales a nivel del maxilar superior y 7 piezas a nivel de la mandíbula; teniendo en cuenta que 1 de ellas es pieza decidua (Tabla 1) (Ilustración 2).

Tabla 1. Piezas dentales presentes en boca separados por cuadrantes.

\begin{tabular}{|c|c|}
\hline \multicolumn{2}{|c|}{ Dientes Erupcionados } \\
\hline 1.21 .1 & $\begin{array}{lll}2.1 & 2.2 & 2.4\end{array}$ \\
\hline $\begin{array}{llll}8.4 & 4.3 & 4.2 & 4.1\end{array}$ & $\begin{array}{lll}3.1 & 3.2 & 3.3\end{array}$ \\
\hline
\end{tabular}

Fuente: Autores.

Ilustración 2. Fotografías intraorales del paciente (a) Fotografía del maxilar superior (b) Fotografía del maxilar inferior.
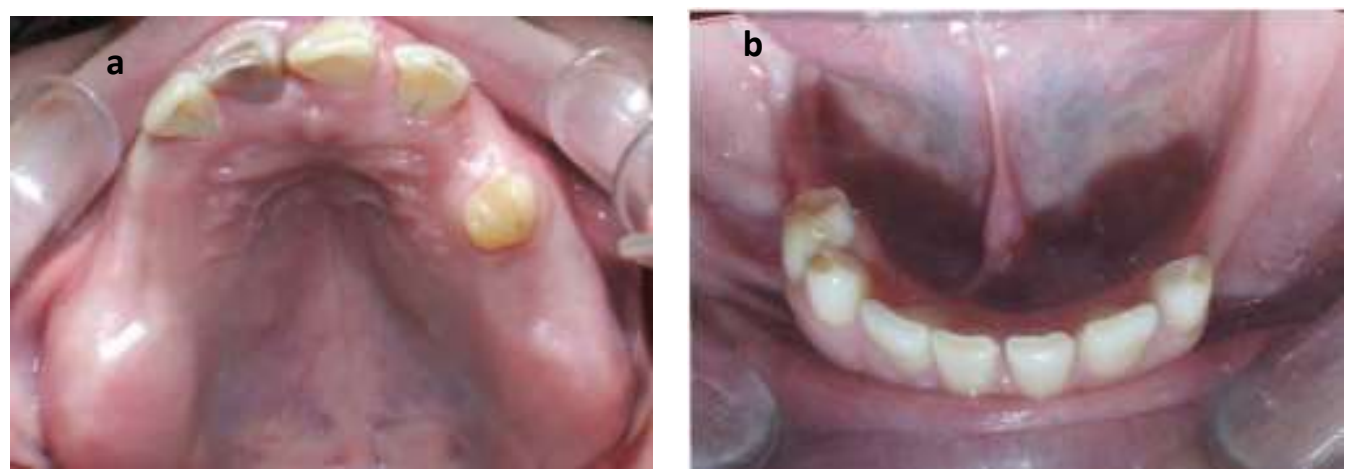

Fuente: Autores. 
Se puede acotar también la ausencia de llaves molares y guías caninas de manera bilateral, en cuanto a la estructura dental se pueden identificar diversas alteraciones morfológicas a nivel coronal y discromía que afecta a la pieza 1.1 (Ilustración 3)

Ilustración 3. Fotografía Intraoral del paciente en oclusión, en la que se observa la discromía de la pieza 1.1.

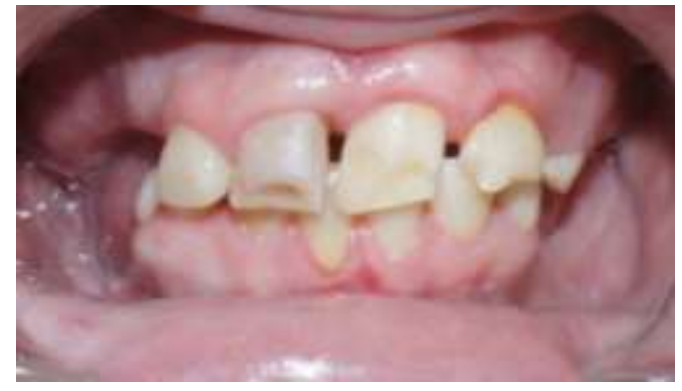

Fuente: Autores.

Al examen radiográfico se observa la presencia de 13 piezas dentarias retenidas, teniendo en cuenta que 3 de estas se ubican a nivel de la mandíbula y las 10 piezas restantes se ubican de manera equitativa en cada hemimaxilar. Se destaca también disminución de la densidad ósea, a nivel de la mandíbula hay la presencia de áreas radiolúcidas no circunscritas en relación con la sínfisis y cuerpo mandibular izquierdo (Ilustración 4).

Luego de revisar los cortes tomográficos realizados, se pudo determinar que las áreas radiolúcidas no correspondía con ninguna actividad patológica, y al indagar datos se relacionó con la enucleación de quistes radiculares previos de los cuales no se tiene un registro fotográfico. También se pudo visualizar la presencia de áreas radiolúcidas bien circunscritas a nivel coronal de las piezas 1.3 y 2.3 compatibles con lesiones quísticas, posiblemente quistes dentígeros (Ilustración 5).

Se toma en consideración la posición de las piezas 1.7, 1.6, 1.4, 1.3, 2.3, 2.4, 2.6, 2.7 que están en contacto directo con la pared posterior, lateral y el piso de los senos maxilares; en tanto que las piezas 3.7, 3.8 y 4.8 se encuentran en relación con el conducto del nervio dentario inferior (Ilustración 4).

Ilustración 4. Radiografía panorámica en la que se puede observar las 13 piezas retenidas y las alteraciones patológicas presentes relacionadas con piezas 1.3 y 2.3 .

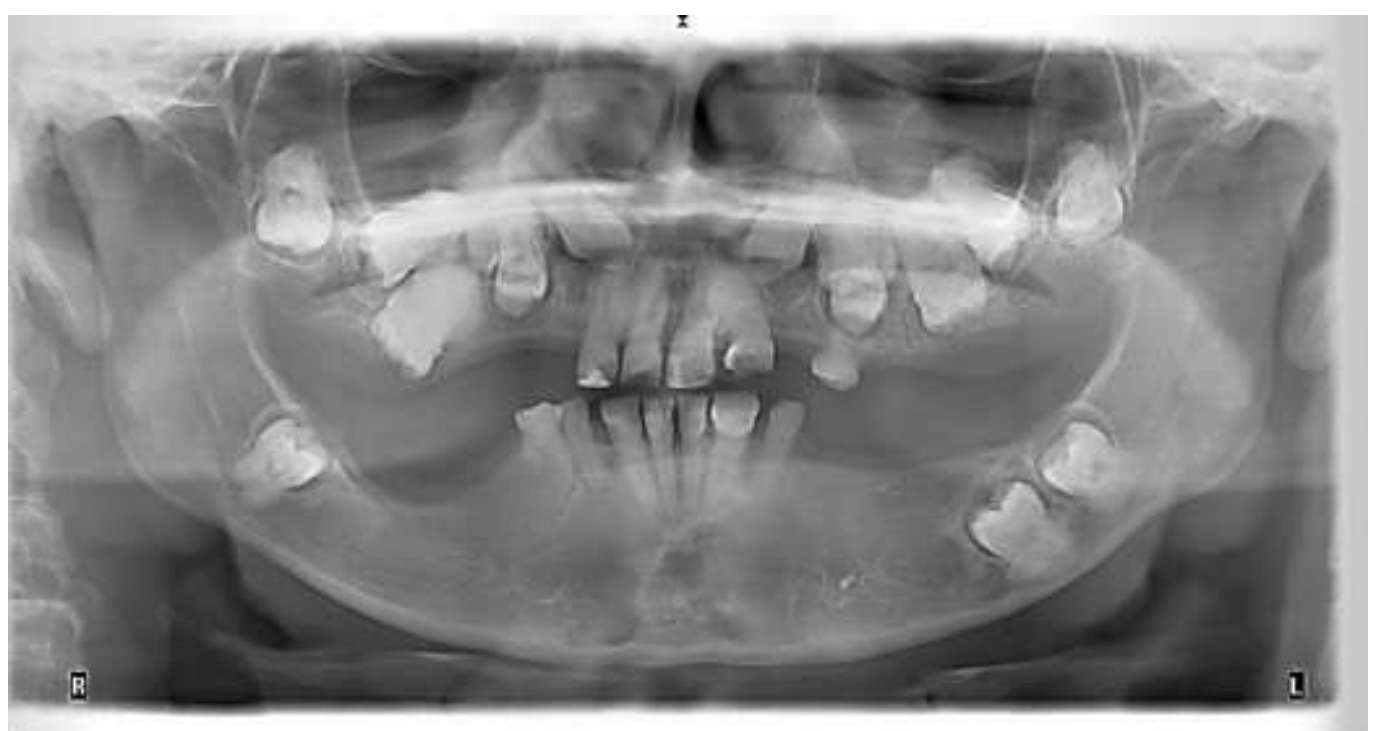

Fuente: Autores. 
Ilustración 5. Diferentes cortes tomográficos en los cuales se aprecia (a) Dimensiones de la pieza 1.3 y la presencia de imagen radiolúcida compatible con formación quística. (b) Ubicación y relación de las piezas con el seno maxilar derecho (c) Dimensiones de la pieza 2.3 y la presencia de imagen radiolúcida compatible con formación quística (d) Ubicación y relación de las piezas con el seno maxilar izquierdo.

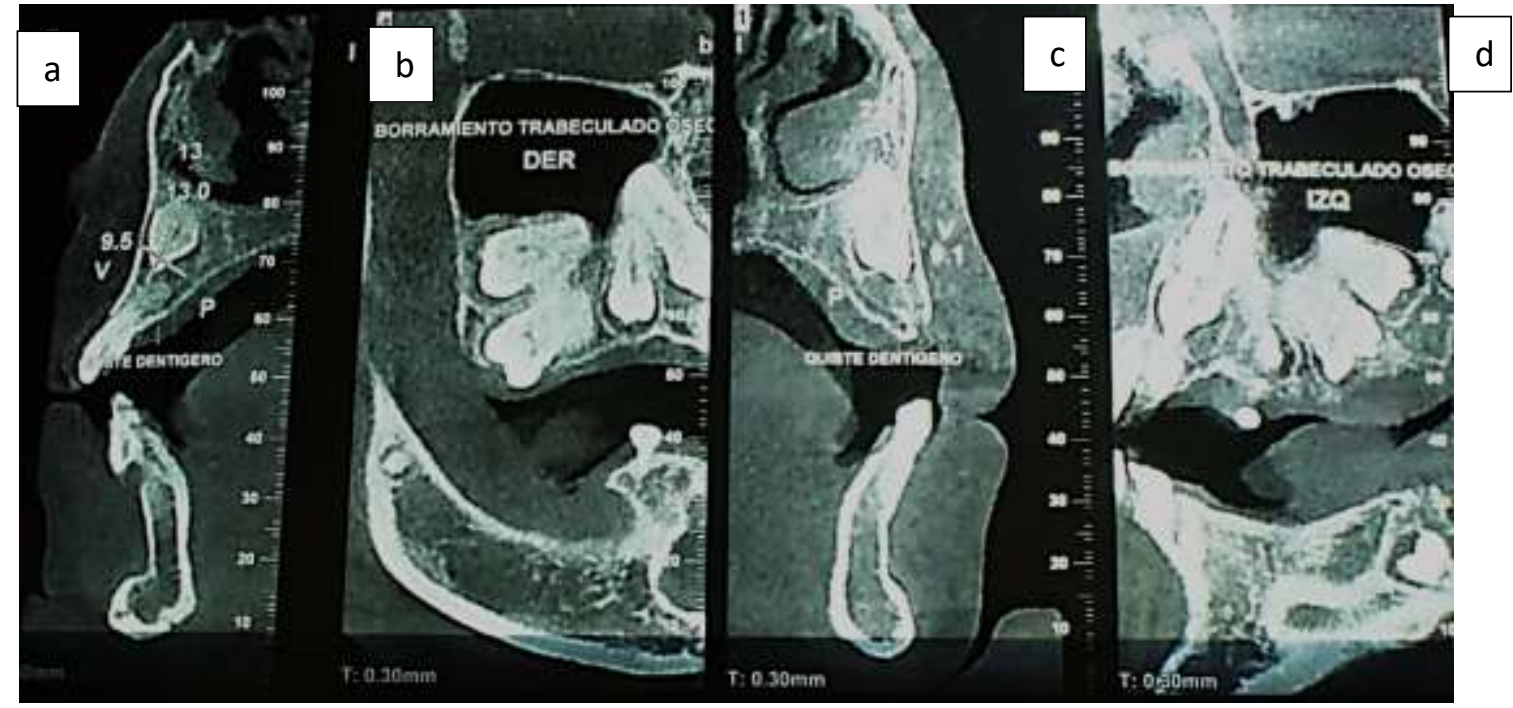

Fuente: Autores.

\section{Discusión}

La erupción dental se define como un proceso de migración que realiza una pieza dental desde el interior de los huesos, tanto la mandíbula y el maxilar, hasta llegar a al plano oclusal dentro de la boca, donde puede cumplir sus funciones (Cahill \& Marks Jr, 1980). Cuando los patrones de crecimiento fallan pueden generar múltiples trastornos eruptivos (Ajith et al., 2014; Choukroune, 2017), que pueden ser denominados de manera errónea por lo que es importante diferenciar entre los términos inclusión, impactación y retención dental primaria o secundaria; ayudándonos para una correcta descripción.

- Inclusión: Presente cuando la erupción dental no se cumple en el tiempo previsto, generando un fracaso del mismo. La razón puede ser la presencia de un obstáculo físico (falta de espacio, tejido blando, duro u otros dientes), por una posición ectópica del diente, o por factores sistémicos. Se observa la pared ósea y su saco pericoronario intacto (Cabrera, Paz, Crugeiras, \& García, 2014).

- Impactación: Se observa cuando la erupción dental sucedía en el tiempo previsto, pero se detiene ya sea por un obstáculo físico o por una posición ectópica del diente. La pared ósea se encuentra perforada y el saco pericoronario puede estar abierto, siendo posible observarse en boca o puede mantenerse submucoso (Cabrera et al., 2014).

- Retención primaria: Se observa una interrupción eruptiva, total o parcial, sin obstáculo físico, además la trayectoria eruptiva y la posición dental es normal; sin embargo, el diente no aparece en la cavidad bucal (Cabrera et al., 2014).

- Retención secundaria: Esta se diferencia de la anterior debido a que el diente en cuestión está presente en la cavidad bucal (Cabrera et al., 2014).

A partir de esta terminología se puede concluir que las piezas descritas en nuestro caso son clasificadas dentro del grupo de retención primaria. Es necesario mencionar que estas piezas dentales pueden estar asociadas a patologías, en este caso podemos observar la presencia de quistes dentígeros; teniendo en consideración que en nuestro medio estas formaciones quísticas se ubican en un 24\% a nivel de la mandíbula (Ishihara, Kamioka, Takano-Yamamoto, \& Yamashiro, 2012). 
Además es importante recalcar que los casos de retención dentaria múltiple son raros y comúnmente se encuentran relacionados directamente con síndromes o enfermedades que se describirán a continuación como: displasia cleidocraneal, síndrome de Gardner, osteopetrosis, hipotiroidismo, Síndrome de Zimmerman- Laband, síndrome de Noonan (Choukroune, 2017; Delavarian et al., 2018; Reddy et al., 2018; Vani et al., 2014).

A continuación, describiremos brevemente ciertas características de importancia que podemos encontrar en pacientes con afectación sindrómica:

Tabla 2. Características importantes de síndromes relacionados con retención dentaria.

\begin{tabular}{|c|c|c|c|}
\hline AUTOR & AÑO & SÍNDROMES & CARACTERÍSTICAS \\
\hline Hassi. J & 2019 & Displasia Cleidocraneal & $\begin{array}{l}\text { 1. Alteraciones a nivel craneal, clavicular, pélvicos, dentales } \\
\text { 2. Braquiocefálicos } \\
\text { 3. Hipoplasia malar } \\
\text { 4. Paladar ojival } \\
\text { 5. Maloclusión dental } \\
\text { 6. Hipoplasia maxilar } \\
\text { 7. Prognatismo mandibular } \\
\text { 8. Retraso en la erupción dental y prolongada exfoliación de } \\
\text { 9. Apiñamiento dentario } \\
\text { 10. Alteraciones en la forma de la corona dental } \\
\text { 11. Múltiples dientes supernumerarios impactados } \\
\text { 12. Ausencia del cemento radicular }\end{array}$ \\
\hline Chimenos, $\mathrm{K}$ & 2019 & Síndrome de Gardner & $\begin{array}{l}\text { 1. Osteomas } \\
\text { 2. Pólipos intestinales y estomacales } \\
\text { 3. Anomalías dentales } \\
\text { a. Múltiples dientes permanentes impactados } \\
\text { b. Dientes supernumerarios } \\
\text { c. Anomalías radiculares } \\
\text { d. Odontomas y cementomas. }\end{array}$ \\
\hline Aranzabal, A & 2019 & Osteopetrosis & $\begin{array}{l}\text { 1. Macrocefalia } \\
\text { 2. Hidrocefalia } \\
\text { 3. Alteraciones metabólicas y hematológicas } \\
\text { 4. Alteraciones en el remodelado óseo } \\
\text { 5. Erupción dentaria retardada } \\
\text { 6. Coronas y raíces malformadas } \\
\text { 7. Disminución del crecimiento del hueso alveolar } \\
\text { 8. Dientes primarios retenidos y permanentes impactados } \\
\text { 9. Engrosamiento anormal de la membrana periodontal }\end{array}$ \\
\hline
\end{tabular}




\begin{tabular}{|c|c|c|c|}
\hline Rodriguez, M & 2014 & Hipotiroidismo & $\begin{array}{l}\text { 1. Cansancio y debilidad } \\
\text { 2. Depresión mental } \\
\text { 3. Crecimiento facial vertical } \\
\text { 4. Disminución de la longitud y el ángulo de la base del cráneo } \\
\text { 5. Labios gruesos y macroglosia } \\
\text { 6. Mordida abierta anterior y dientes anteriores en abanico. }\end{array}$ \\
\hline Estevez, $\mathrm{N}$ & 2005 & $\begin{array}{l}\text { Síndrome de } \\
\text { Zimmerman- Laband }\end{array}$ & $\begin{array}{l}\text { 1. Fibromatosis gingival a nivel mandibular y puede progresar } \\
\text { hasta paladar blando. } \\
\text { 2. Erupción dentaria fallida o presencia de dientes } \\
\text { supernumerarios. } \\
\text { 3. Anormalidades esqueléticas como displasia de falanges } \\
\text { 4. Hepatomegalia y esplenomegalia. } \\
\text { 5. Hiperlaxitud articular. } \\
\text { 6. Cataratas bilaterales o defectos de refracción. } \\
\text { 7. Retardo mental profundo. }\end{array}$ \\
\hline Uloopy, KS & 2015 & Síndrome de Noonan & $\begin{array}{l}\text { 1. Paladar arqueado } \\
\text { 2. Afectación cardíaca: miocardiopatía hipertrófica } \\
\text { 3. Múltiples defectos esqueléticos } \\
\text { 4. Cuello palmeado } \\
\text { 5. Retraso mental } \\
\text { 6. Maloclusión dental } \\
\text { 7. Dificultades de articulación } \\
\text { 8. Micrognacia. } \\
\text { 9. Quistes mandibulares }\end{array}$ \\
\hline
\end{tabular}

(Hassi, Izquierdo, \& Narea, 2019) (Chimenos-Küstner, Pascual, Blanco, \& Finestres, 2005) (Aranzábal-Alegría, Espinoza-Chiong, Benites-Gamboa, \& Aguirre-Retamozo, 2019) (Rodríguez, García, \& Flores, 2014) (Estévez \& Pedraza, 2005) (Uloopi, Madhuri, Gopal, Vinay, \& Chandrasekhar, 2015)

Fuente: Autores.

Luego de realizar esta recapitulación sobre los síndromes con mayor prevalencia de presentar dientes retenidos y sus características clínicas se realizó una correlación tomando en cuenta lo observado en el paciente, en el cual se pudo detectar ciertas similitudes con la displasia cleidocraneal, ya que en su cavidad oral podemos encontrar la presencia de hiperdoncia en los dientes anteriores, mala alineación dental, erupción tardía, retención de piezas definitivas, anomalías anatómicas de las coronas dentales, hipoplasia del esmalte, y quistes dentígeros, dichos aspectos se asemejan a los descrito por Hassi y cols. en el año 2019 (Hassi et al., 2019).

Sin embargo, no es posible diagnosticar al paciente con dicha patología pues carecemos de pruebas genéticas, es decir, pruebas de cariotipo que nos ayude a identificar y aseverar nuestras descripciones realizadas. 
Ilustración 6. Revisión bibliográfica sobre retención dentaria.

\begin{tabular}{|c|c|c|c|c|c|c|}
\hline Autor & Año & Sexo & Edad & $\begin{array}{l}\text { Lado de } \\
\text { ubicación }\end{array}$ & Piezas & Patología Asociada \\
\hline Delavarian, M & 2018 & $\begin{array}{l}\text { Masculino } \\
\text { Femenino }\end{array}$ & 17 Y 16 & Bimaxilar & $\begin{array}{l}22 \text { Retenciones- } 9 \\
\text { Piezas } \\
\text { supernumerarias } \\
9 \text { Retenciones }\end{array}$ & $\begin{array}{l}\text { Paciente sin asociación } \\
\text { aparente }\end{array}$ \\
\hline Reddy, G & 2018 & Femenino & 16 & Bimaxilar & 16 Retenciones & $\begin{array}{ll}\text { Paciente hijo de } \\
\text { matrimonio } \\
\text { consanguíneo } \\
\text { positivo } \\
\text { Hipoparatiroidismo }\end{array}$ \\
\hline Vani, S & 2014 & Masculino & 21 & Bimaxilar & 29 Retenciones & $\begin{array}{l}\text { Paciente sin asociación } \\
\text { aparente }\end{array}$ \\
\hline Sujatha, G & 2012 & Masculino & 17 & Bimaxilar & 19 Retenciones & $\begin{array}{l}\text { Presencia de barrera } \\
\text { física condujo a la } \\
\text { impactación }\end{array}$ \\
\hline Al Rhaka, D & 2018 & Masculino & 34 & Bimaxilar & 19 Retenciones & $\begin{array}{l}\text { Paciente sin asociación } \\
\text { aparente }\end{array}$ \\
\hline Casas Acosta, J & 2019 & Femenino & 12 & Unimaxilar & 2 Retenciones & $\begin{array}{l}\text { Paciente } \sin \text { asociación } \\
\text { aparente }\end{array}$ \\
\hline Ishihara, Y & 2012 & Masculino & 13 & Unimaxilar & 2 Retenciones & $\begin{array}{l}\text { Paciente } \\
\text { quistes } \\
\text { asociados } \\
\text { (Ishihara et al., 2012) }\end{array}$ \\
\hline López, M & 2017 & Femenino & 10 & Bimaxilar & 5 Retenciones & $\begin{array}{l}\text { Paciente sin asociación } \\
\text { aparente }\end{array}$ \\
\hline Moturi, K & 2018 & Femenino & 19 & Bimaxilar & 13 Retenidos & $\begin{array}{l}\text { Paciente sin asociación } \\
\text { aparente }\end{array}$ \\
\hline
\end{tabular}

Fuente: (Al Rakah, Al Muhanna, \& El Ghazali, 2018; Casas Acosta et al., 2020; Delavarian et al., 2018; Ishihara et al., 2012; López \& Valdivia, 2017; Moturi \& Kaila, 2018; Reddy et al., 2018; Sujatha et al., 2012; Vani et al., 2014).

Por dicha razón, realizamos una revisión sobre distintos autores que presentan casos de múltiples retenciones dentarias en las cuales se observó mayor prevalencia en el género masculino, siendo distinto a lo previamente observado en los estudios de Conley, Sreevedi y Manne. (Ajith et al., 2014; Conley et al., 2007; Manne et al., 2012). En relación al rango de edad prevalente tras la revisión realizada fue entre 12-34 años, además se observó mayormente retenciones de carácter bimaxilar y por lo general estaban involucradas más de dos piezas por lo cual se consideraban como múltiples. Tras la revisión pudimos visualizar la asociación directa con enfermedades sistémicas y aunque sigue siendo muy pocos los autores que reportan casos de retención dental múltiple idiopática, se abre la puerta para visibilizar la existencia de dicha condición y buscar más información que permita conocer a detalle sobre diversos casos que se pueden presentar a nivel de Latinoamérica y generar estudios epidemiológicos posteriores.

\section{Conclusión}

La presencia de múltiples piezas retenidas se describe como una patología poco común, que puede tener asociación con factores tanto locales como sistémicos. Esto afecta en su mayoría al sexo femenino, además se pueden observar formaciones quísticas que acompañen a dichas piezas. Por ende, es fundamental que, tras realizar una correcta anamnesis, un análisis extra e intraoral detallado, se acompañe de estudios imagenológicos que nos permitan llegar a un correcto diagnóstico y un buen plan de tratamiento. Para la determinación de dicha patología se recomienda realizar más estudios y análisis sobre causas sindrómicas y no sindrómicas que nos permitan conocer una base sólida sobre un posible origen genético. 


\section{Referências}

Ajith, S. D., Shetty, S., Hussain, H., Nagaraj, T., \& Srinath, M. (2014). Management of multiple impacted teeth: A case report and review. Journal of international oral health: JIOH, 6(3), 93.

Al Rakah, D., Al Muhanna, H., \& El Ghazali, S. (2018). Idiopathic Multiple Impacted Teeth: A Case Report. Journal of Medical Cases, 9(2), 41-44.

Aranzábal-Alegría, G., Espinoza-Chiong, C., Benites-Gamboa, D., \& Aguirre-Retamozo, L. (2019). Osteopetrosis maligna infantil: a propósito de un caso y revisión de la literatura. Revista de la Facultad de Medicina Humana, 19(4), 126-130.

Bedoya, M. M., \& Park, J. H. (2009). A review of the diagnosis and management of impacted maxillary canines. The Journal of the American Dental Association, 140(12), 1485-1493.

Cabrera, A., Paz, J., Crugeiras, V., \& García, M. (2014). Actualización de conceptos en relación a los trastornos eruptivos. Puesta al día. Gaceta dental: Industria y profesiones, 258, 136-153.

Cahill, D. R., \& Marks Jr, S. C. (1980). Tooth eruption: evidence for the central role of the dental follicle. Journal of Oral Pathology \& Medicine, 9(4), 189200 .

Casas Acosta, J. E., Sardiña Valdés, M., del Busto Chinea, M., Peñate Sardiña, C. O., Peñate Sardiña, D., \& López Puig, J. (2020). Retenciones múltiples. Reporte de un caso. Revista Médica Electrónica, 42(3), 1900-1910.

Conley, R. S., Boyd, S. B., Legan, H. L., Jernigan, C. C., Starling, C., \& Potts, C. (2007). Treatment of a patient with multiple impacted teeth. The Angle Orthodontist, 77(4), 735-741.

Chimenos-Küstner, E., Pascual, M., Blanco, I., \& Finestres, F. (2005). Poliposis familiar hereditaria y síndrome de Gardner: Aportación de la exploración odontoestomatológica a su diagnóstico y descripción de un caso. Medicina Oral, Patología Oral y Cirugía Bucal (Ed. impresa), 10(5), 402-409.

Choukroune, C. (2017). Tooth eruption disorders associated with systemic and genetic diseases: clinical guide. Journal of Dentofacial Anomalies and Orthodontics, 20(4), 402.

Delavarian, M., Delavarian, F., \& Imani, M. M. (2018). Non-syndromic multiple impacted teeth: two case reports. Biomedical Research and Therapy, 5(11), 2798-2801.

Estévez, N. S. M., \& Pedraza, M. (2005). Síndrome de Zimmermann Laband. Universitas Médica, 46(4), $147-149$.

Hassi, J., Izquierdo, C., \& Narea, G. (2019). Displasia Cleidocraneal: Manejo ortodóncico y quirúrgico, seguimiento a 10 años. Revista clínica de periodoncia, implantología y rehabilitación oral, 12(2), 109-112.

Hernandez, A. A. (2018). Metodología de la investigación científica. In e-conversion - Proposal for a Cluster of Excellence.

Ishihara, Y., Kamioka, H., Takano-Yamamoto, T., \& Yamashiro, T. (2012). Patient with nonsyndromic bilateral and multiple impacted teeth and dentigerous cysts. American journal of orthodontics and dentofacial orthopedics, 141(2), 228-241.

López, M. L. V., \& Valdivia, I. G. (2017). Manejo Ortodontico de paciente con inclusiones dentales múltiples y transposición dental. Revista Mexicana de Ortodoncia, 5(1), 27-34.

Manne, R., Gandikota, C., Juvvadi, S. R., Rama, H. R. M., \& Anche, S. (2012). Impacted canines: Etiology, diagnosis, and orthodontic management. Journal of pharmacy \& bioallied sciences, 4(Suppl 2), S234.

Moturi, K., \& Kaila, V. (2018). Management of non-syndromic multiple impacted teeth with dentigerous cysts: a case report. Cureus, 10(9).

Reddy, G. S., Chalapathi, K., Reddy, D. S., Rana, S., Kalyan, M., Kartheeki, B., \& Nayyar, A. S. (2018). Multiple impacted permanent teeth, an indicator for early detection of hypoparathyroidism: A rare case report. Journal of family medicine and primary care, 7(5), 1096.

Rodríguez, M. E. R., García, M. A. M., \& Flores, I. S. (2014). Hipotiroidismo congénito y sus manifestaciones bucales. Revista odontológica mexicana, 18(2), $132-137$.

Sosa Rosales, M. d. 1. C., Garrigó Andreu, M., Sardiña Alayón, S., Gispert Abreu, E., Valdés García, P., \& Legón Padilla, N. (2003). Guías prácticas de Estomatología. La Habana: Editorial Ciencias Médicas, 13-75.

Sujatha, G., Sivapathasundharam, B., Sivakumar, G., Nalinkumar, S., Ramasamy, M., \& Prasad, T. S. (2012). Idiopathic multiple impacted unerupted teeth: Case report and discussion. Journal of oral and maxillofacial pathology: JOMFP, 16(1), 125.

Uloopi, K., Madhuri, V., Gopal, A., Vinay, C., \& Chandrasekhar, R. (2015). Case report: Multiple unerupted permanent teeth associated with Noonan syndrome. Annals of medical and health sciences research, 5(4), 317-320.

Uslu, O., Akcam, M. O., Evirgen, S., \& Cebeci, I. (2009). Prevalence of dental anomalies in various malocclusions. American Journal of Orthodontics and Dentofacial Orthopedics, 135(3), 328-335.

Vani, S., Nooney, A., Raju, K. S., \& Hemadri, M. (2014). Idiopathic multiple unerupted permanent teeth: A rare case report. Journal of Dr. NTR University of Health Sciences, 3(4), 283.

Yeman, O. (2003). Incisivos centrales superiores retenidos. Rev Soc Odont Argentina, 6(2), 21-26. 